\title{
Aphallia Associated with Anorectal Malformation
}

"Ruben Blachman-Braun, ${ }^{1}$ Cinthia Galvez, ${ }^{2}$ Christian Guevara, ${ }^{2}$ Raquel Quintanilla, ${ }^{2}$ Alireza Alam ${ }^{2}$

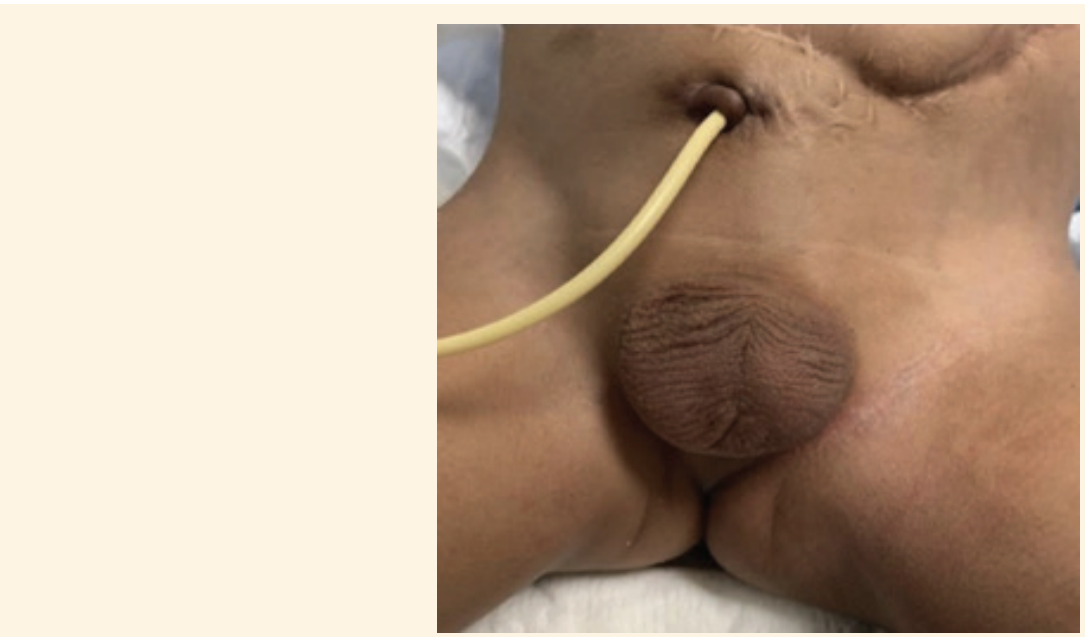

Figure 1: Frontal clinical image of the lower abdomen showing total absence of penis and normal scrotum with both descended testicles. A suprapubic tube with a $10 \mathrm{Fr}$ Foley catheter draining urine and old surgical scars on the left hemiabdomen can also be observed.

\section{A} 3-YEAR-OLD HISPANIC MALE INFANT WAS admitted to the paediatric urology department at a children's hospital in Florida, USA, with a febrile urinary tract infection and constipation. The patient's perinatal history included $\mathrm{C}$-section delivery due to premature membrane rupture $(\mathrm{G} 1, \mathrm{P} 1)$ at the gestational age of 36 weeks. The patient was born in Nicaragua with anorectal malformation and without fistula, aphallia and a perineal urethra. The infant underwent colostomy during the neonatal period followed by posterior-sagittal anorectoplasty, perineal urethral closure and suprapubic catheter (SPC) placement. Six months following colostomy closure at two years of age, the patient arrived at the current institution in the USA. On physical examination, an SPC to gravity in the suprapubic region and normally descended testicles along with an absence of phallus were observed. The neo-anus was well-located and there were no abnormalities in the sacral region. There were also no deformities in the extremities [Figure 1]. The renal and bladder ultrasound showed malrotated kidneys and magnetic resonance imaging showed no visualisation of the corpora cavernosa or corpus spongiosum, normal sacrum and spinal cord [Figure 2]. Following multidisciplinary evaluation and shared decision-making with the patient and the family, the current team will consider performing a neophalloplasty in the future. Informed consent was obtained from the patient's mother for the publication of the case and the associated images.

\section{Comment}

Aphallia is an extremely rare sporadic genitourinary malformation with less than 100 cases reported worldwide..$^{1-3}$ It is defined as an absent phallus with complete absence of the two corpora cavernosa and the corpus spongiosum. In addition, it is usually associated with an ectopic and short urethral opening to the rectum or anus and a well-formed scrotum with both testes descended. Aphallia may also occur in female patients since it is the absence of corporal tissue that is considered the defining factor of the aphallia, although this is less common and more difficult to diagnose. ${ }^{4}$ During the fourth week of gestation, a mesenchymal prominence within the cloacal membrane proliferates to form the genital tubercle, which then develops into a penis or clitoris. It is hypothesised that aphallia results from the developmental failure or absence of the genital tubercle. Animal models suggest that abnormalities in the sonic hedgehog (shh) genecritical in normal bladder and bowel development- 


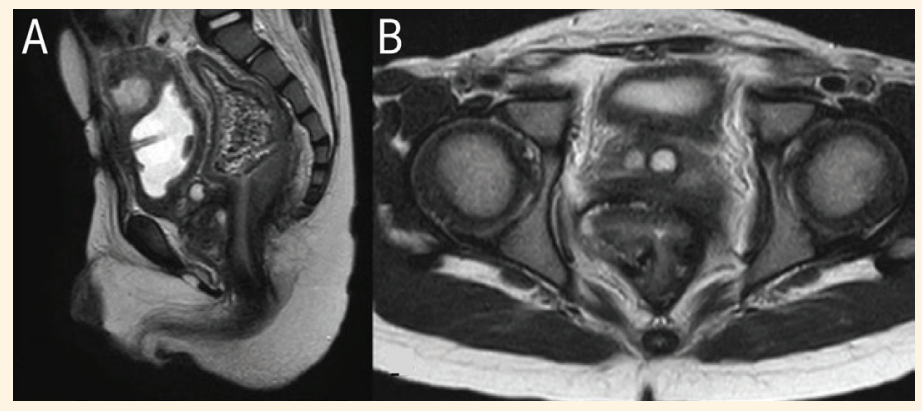

Figure 2: A: Magnetic resonance imaging (MRI) scan in a sagittal view of the lower abdomen and pelvis showing an under distended bladder, a suprapubic catheter and no cavernosa bodies, corpus spongiosum or urethra. The sacrum and spinal cord show no abnormalities. B: MRI scan in an axial view showing no cavernosa bodies, corpus spongiosum or urethra.

may be linked to aphallia. ${ }^{4}$ Shh knockout mice demonstrate genital tubercle agenesis, most likely through impaired signalling of the fibroblast growth factor. These models also suggest that impaired retinoic acid signalling within the shh molecular pathway may also be the cause of genital tubercle agenesis via inhibited vasculogenesis. ${ }^{4}$ Furthermore, abnormalities in the shh pathway are also critical in the explanation of the high incidence of concomitant anorectal malformations seen in aphallia. It is possible that polymorphism located in $9 \mathrm{qh}+$ might be part of the underlying aetiology as well. ${ }^{4,5}$ In over $50 \%$ of patients, aphallia presents as a symptom of a broader spectrum of congenital malformations; renal, urinary tract and anorectal malformations are frequently associated with each other. However, aphallia may also present as an isolated disorder, such as in cases where the genitourinary system has a normal configuration. ${ }^{2}$

Most children with this disorder of sex development have 46,XY karyotype; however, it may also be seen in patients with congenital adrenal hyperplasia and 46,XX karyotype. The differential diagnoses include concealed penis, intra-uterine amputation of the penis or 46,XY disorders of sex differentiation. Aphallia may be classified in numerous ways: using Skoog's anatomical classification, based on the position of the ectopic urethra in relation to the anus and the number of associated anomalies. ${ }^{2}$ Using Skoog's anatomical classification, aphallia may be subclassified as pre-sphincteric, post-sphincteric or urethral atresia, with this last being the least common (12\% of cases) and uniformly fatal. Evans' prognostic classification, solely based on the presence or absence of major associated anomalies, may be used as well. ${ }^{2}$

The management approaches are divided into short, intermediate and long-term treatment phases. While short-term treatment involves dealing with any life-threatening complications and associations of aphallia that may present in the neonatal period, intermediate-term treatment typically consists of division of the urethrorectal fistula with perineal urethrostomy. Finally, long-term treatment commits to the creation of a neophallus that will allow the patient to appear outwardly male and be able to void while standing up. ${ }^{2}$

In conclusion, aphallia is an extremely rare sporadic genitourinary malformation. It is characterised by the absence of the two corpora cavernosa and the corpus spongiosum, usually the scrotum is well-formed, the urethra is ectopic, short and frequently opens in the rectum or anus. It is hypothesised that aphallia results from the absence or developmental failure of the genital tubercle and the exact aetiology still need to be elucidated. A multidisciplinary evaluation and shared decision-making with the patient and the family are needed to provide optimal outcomes.

\section{AUTHORS' CONTRIBUTION}

$\mathrm{AA}$ and $\mathrm{RB}-\mathrm{B}$ conceptualised and designed the work. RB-B, CiG, ChG, RQ and AA were involved in data acquisition, analysis and interpretation. All authors contributed equally to drafting the manuscript and approved the final version of this manuscript.

\section{References}

1. Talebpour Amiri F, Nasiry Zarrin Ghabaee D, Naeimi RA, Seyedi SJ, Mousavi SA. Aphallia: Report of three cases and literature review. Int J Reprod Biomed 2016; 14:279-84. https:// doi.org/10.29252/ijrm.14.4.279.

2. Gabler T, Charlton R, Loveland J, Mapunda E. Aphallia: a review to standardize management. Pediatr Surg Int 2018; 34:813-21. https://doi.org/10.1007/s00383-018-4271-z.

3. OliveiraDE, daCruzML,LiguoriR, GarroneG,LeslieB,OttoniSL, et al. Neophalloplasty in boys with aphallia: A systematic review. J Pediatr Urol 2016; 12:19-24. https://doi.org/10.1016/j. jpurol.2015.10.003.

4. Friedman AA, Zelkovic PF, Reda EF, Franco I, Palmer LS. Male and female aphallia associated with severe urinary tract dysplasia. J Pediatr Urol 2016; 12:268.e1-7. https://doi. org/10.1016/j.jpurol.2016.04.040.

5. Qiang S, Li FY, Zhou Y, Yuan Y, Li Q. Congenital absence of the penis (aphallia): A rare case report. Medicine (Baltimore) 2019; 98:e15129. https://doi.org/10.1097/MD.0000000000015129. 